\title{
Treino prévio reduz o tempo de execução de tarefas visuo-espaciais em ambiente virtual
}

\author{
Previous training reduces the execution time of visuo-spacial tasks in virtual environment \\ Adriano Conrado Rodrigues ${ }^{1}$, Carla da Silva Santana ${ }^{2}$, Roberta de Medeiros 3 , \\ Sandra Regina Alouche ${ }^{4}$
}

\begin{abstract}
RESUMO
Objetivo. Avaliar a influência da adaptação prévia de indivíduos sadios a diferentes situações de interação em ambiente computacional, no desempenho de tarefas visuo-espaciais. Método. Trinta voluntários saudáveis, 15 homens, com idade média de 25,3 \pm 3,3 anos, foram divididos em três grupos: o primeiro, considerado controle, não foi adaptado a nenhum dos ambientes propostos; o segundo grupo foi adaptado ao ambiente fechado (estável e previsível) e o terceiro foi adaptado ao ambiente aberto (imprevisível) "A". A tarefa proposta era o deslocamento em dois ambientes abertos "B" e "C" (labirinto). As variáveis dependentes Tempo e Erro foram consideradas para a análise. Resultados. Foi observado que durante a fase de adaptação, na variável Tempo, os grupos apresentaram uma progressiva melhora no desempenho de cada tarefa $(p=0,0036)$. O grupo adaptado ao ambiente aberto "A" mostrou uma tendência a ser mais rápido na execução das tarefas dos ambientes abertos "B" e "C", em relação ao grupo adaptado ao ambiente fechado $(\mathrm{p}=0,068)$. Conclusão. Este estudo sugere que indivíduos adaptados à execução de tarefas visuoespaciais que envolvam situações desconhecidas e não orientadas, apresentam uma tendência a um melhor desempenho do tempo nestas tarefas, quando comparados a indivíduos adaptados em situações estáveis e orientadas.
\end{abstract}

Unitermos: Análise e Desempenho de Tarefas. Aprendizagem. Meio Ambiente.

Citação: Rodrigues AC, Alouche SR, Medeiros R, Santana C.S. Treino prévio reduz o tempo de execução de tarefas visuo-espaciais em ambiente virtual.

\section{SUMMARY}

Objective. To evaluate the influence of previous adaptation to different computational environments in visuo-spacial tasks performance of healthy individuals. Method. Healthy volunteers $(\mathrm{n}=30), 15$ male, mean age $25.3 \pm 3.3$ years, were divided in three groups: the first group, considered control, was not adapted to the proposed environments; the second group was adapted to a closed environment (stable and expected), and the third group was adapted to an open environment " $\mathrm{A}$ " (unexpected). The proposed task was to go through two open environments "B" and "C" (maze). The dependent variables Time and Error were considered for the analysis. Results. It was observed that during the adaptation phase, in the Time variable, the groups presented a progressive improvement in the performance to each task $(\mathrm{p}=0.0036)$. The group adapted in the "A" open environment, showed a tendency to be faster in the execution of " $\mathrm{B}$ " and " $\mathrm{C}$ " open environments tasks, than the group adapted in the closed environment $(\mathrm{p}=0.068)$. Conclusion. The study suggests that subjects adapted to visuo-spacial tasks execution involving unknown and no guided situations, present a tendency to a better time performance in these tasks when compared to subjects adapted in fixed and guided situations.

Keywords: Task Performance and Analysis. Learning. Environment.

Citation: Rodrigues AC, Alouche SR, Medeiros R, Santana CS. Previous training reduces the execution time of visuo-spacial tasks in virtual environment.
Trabalho realizado na Universidade Bandeirante de São Paulo. 1. Terapeuta Ocupacional, Mestre em Ciências da Reabilitação Neuro-motora - UNIBAN, Professor titular da Universidade de Sorocaba. 2. Terapeuta Ocupacional, Doutora em Psicologia Escolar e do Desenvolvimento - USP, Professora Titular USP - FMRP.

3. Bióloga, Doutora em Fisiologia Comparada - UNESP, Professora Titular UNISC.

4. Fisioterapeuta, Doutora em Psicologia (Neurociências e Comportamento) - USP, Docente do Programa de Mestrado em Fisioterapia da Universidade Cidade de São Paulo.
Endereço para correspondência: R Francisca Júlia 618/11 02403-011 São Paulo, SP

Fone: (11) 7605-7092

Recebido em: 08/06/08 Revisado em: 09/06/08 a 23/09/08

Aceito em: 24/09/08

Conflito de interesses: não 


\section{INTRODUÇÃO}

A função primária da capacidade de aprender é desenvolver comportamentos adaptados a um ambiente que muda constantemente, permitindo que realizemos nossas atividades da vida diária ${ }^{1}$.

Para estar adequado, o movimento deve satisfazer as condições ambientais. O movimento de pegar um copo deve estar adaptado aos fatores ambientais da forma do copo, distância do indivíduo e volume do líquido. $\mathrm{O}$ objeto, neste caso o copo, determina a organização espacial do movimento ${ }^{2}$.

As abordagens ecológica e dinamicista do controle motor procuram explicar a relação de um indivíduo com seu ambiente de interação. Esta relação aparece como determinante na organização e controle dos movimentos.

Para os autores destas teorias, a ação é mais do que o resultado da ativação de um grupo de células nervosas corticais, como preconizado pelas teorias representacionalistas. Pode-se dizer que o movimento é primariamente determinado pela ação, e seu controle dependerá da integração entre vários parâmetros e sistemas (físicos, biológicos e culturais). Assim, não existem comportamentos específicos para a execução de uma tarefa, e sim, padrões decorrentes da relação entre estes sistemas ${ }^{3}$.

A teoria dinamicista não preconiza a necessidade de representações, prescrições e programas motores. A coordenação do movimento aparece como consequência de uma auto-organização em busca de um estado de equilíbrio, permitindo que o indivíduo se adapte a uma determinada situação ${ }^{3-5}$.

Esta adaptação pode dar-se em um sistema aberto ou fechado. $\mathrm{O}$ sistema aberto proporciona ao indivíduo uma interação constante com o ambiente, que inclui condições de desequilíbrio, exigindo a auto-organização do sistema. Já no sistema fechado, o ambiente é previsível e estável ${ }^{6}$.

A partir desta interação entre o indivíduo e o ambiente durante atividades funcionais cotidianas, e da estreita ligação entre percepção e ação na execução destas atividades, têm-se que a relação entre informações do ambiente e a capacidade individual de captar e processar estas informações podem ser determinantes na avaliação do desempenho e do controle motor. Assim, a efetividade na execução da ação será limitada pela habilidade de auto-análise do indivíduo e análise do ambiente em que a ação ocorrerá, determinando problemas ou variáveis a serem enfrentados. A capacidade de gerar e controlar solu- ções para estas variáveis, determinará o desempenho do indivíduo na realização de suas atividades ${ }^{4,7-11}$.

Este estudo tem por objetivo verificar a influência da adaptação prévia de indivíduos sadios no desempenho de tarefas visuo-espaciais, realizadas em ambiente computacional.

Assim, esperamos que indivíduos adaptados à execução de tarefas visuo-espaciais que envolvam situações desconhecidas e não orientadas (ambiente aberto) apresentem melhor desempenho nestas tarefas quando comparados a indivíduos adaptados a situações estáveis e orientadas (ambiente fechado).

\section{MÉTODO}

\section{Casuística}

Trinta indivíduos sadios (voluntários) foram identificados como normais em relação ao comportamento motor, por meio de um questionário, abordando pontos como o hábito em jogos de computador, alteração relacionada a visão, alteração física ou deficiência física, alteração mental ou deficiência mental, uso de medicação, alteração do sono (insônia), aspectos emocionais (depressão, ansiedade, apatia, stress), tremor ou algum problema relacionado a coordenação do movimento, déficit de equilíbrio, uso de álcool, drogas ou entorpecentes, alteração na audição, independência nas atividades cotidianas (trabalho, lazer e auto-manutenção), dor, dificuldade de aprendizado e memória. Foram excluídos indivíduos que referiram qualquer alteração nos pontos acima citados, que pudesse interferir em suas atividades cotidianas, assim como indivíduos que referiram experiência prévia habitual ou diária com jogos em ambiente computacional, o que poderia interferir no resultado final da pesquisa, segundo os objetivos propostos.

A faixa etária da amostra foi estabelecida entre 20 e 30 anos (25,3 \pm 3,292 anos), com indivíduos de ambos os sexos, sendo 15 homens e 15 mulheres, destros, e com o segundo grau de formação escolar completo.

Todos assinaram o Termo de Consentimento, concordando formalmente em participar do estudo. Os procedimentos descritos neste estudo foram aprovados pelo comitê de ética da Universidade Bandeirante de São Paulo - UNIBAN, segundo o protocolo No. 006/2004, datado de 18/06/2004.

Local

Os experimentos foram desenvolvidos em uma sala de $9 \mathrm{~m}^{2}$ (3 x 3m), com isolamento acús- 
tico parcial e iluminação artificial, na Universidade Bandeirante de São Paulo. Nesta sala, o voluntário foi adequadamente posicionado sentado em frente a uma mesa, sobre a qual estava um computador e um joystick utilizados para os testes.

\section{Material}

Para a coleta de dados e análise do comportamento motor, foram desenvolvidos quatro ambientes computacionais em forma de labirinto, utilizando-se o software Game Maker $5^{\circledR}$ - copyright $^{\odot}$ 2000-2003 (Figura 1).

Os participantes (representados por um ícone controlado por joystick) foram orientados a interagir, no menor tempo, e com o menor índice de erros possíveis.

O primeiro ambiente reproduziu as características de um ambiente fechado (previsível e estável); e os ambientes "A", "B" e "C" reproduziram as características de um ambiente aberto (imprevisível).

O "labirinto" que representou o ambiente fechado se caracterizou por não apresentar passagens marginais, passagens ocultas e paredes ocultas; o ícone vermelho representa o participante; o ícone

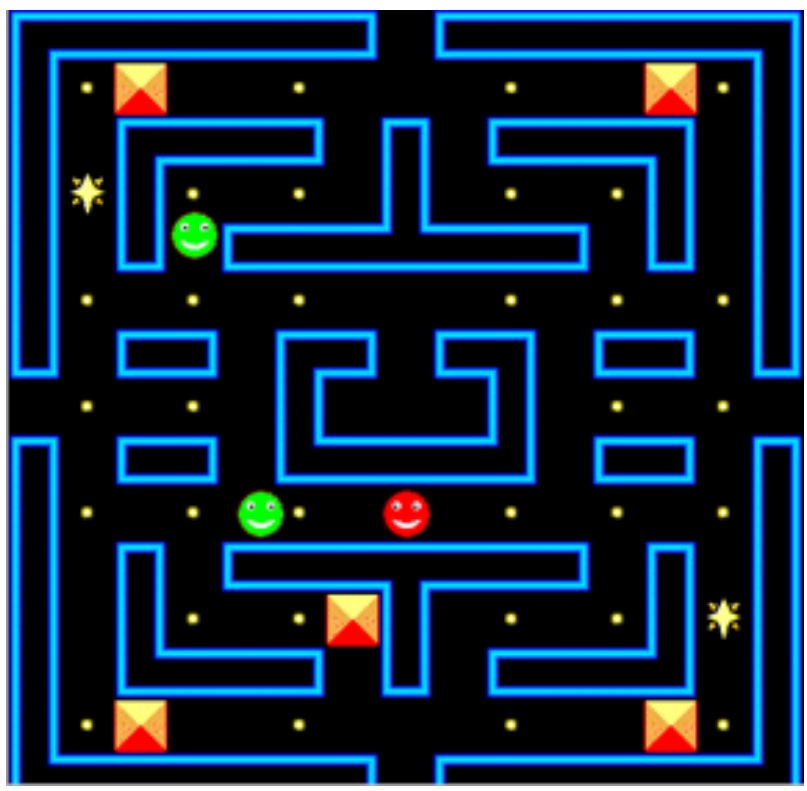

Figura 1. Labirinto: Ambiente fechado - labirinto caracterizado por não apresentar passagens marginais, passagens ocultas e paredes ocultas; o ícone vermelho representa o participante; o ícone verde representa a pista a ser evitada (imóvel); os ícones alaranjados e amarelos representam pistas a serem captadas (estáticas/presentes desde o início do jogo). Ambiente aberto - labirinto caracterizado por apresentar 4 passagens marginais e 2 paredes ocultas; o ícone vermelho representa o participante; ícones verdes representam pistas a serem evitadas (móveis/direções aleatórias); ícones alaranjados e amarelos representam pistas a serem captadas (estáticas/aparecendo após o início do jogo); ícones estrela, quando captados, permitem interação com as pistas representadas pelos ícones verdes, por período específico de tempo, sem caracterizar erro. verde representa a pista a ser evitada (imóvel); cada encontro com esta pista representa 1 erro cometido; os ícones alaranjados e amarelos representam pistas a serem captadas (estáticas/presentes desde o início do jogo).

O "labirinto" que representou o ambiente aberto "A" apresenta 4 passagens marginais e 2 paredes ocultas; o ícone vermelho representa o participante; ícones verdes representam pistas a serem evitadas (móveis/direções aleatórias); cada encontro com esta pista representa 1 erro cometido; ícones alaranjados e amarelos representam pistas a serem captadas (estáticas/aparecendo após o início do jogo); ícones estrela, quando captados, permitem interação com as pistas representadas pelos ícones verdes, as quais tornam-se azuis por um breve período de tempo (período de interação sem caracterizar erro), retomando as características do estado inicial após alguns segundos.

Os "labirintos" representados pelos ambientes abertos "B" e "C" se apresentam como o ambiente Aberto "A", mas com nova configuração de cores, disposição das pistas, passagens e paredes ocultas.

Os ambientes foram apresentados e os dados coletados por meio de um computador com microprocessador Pentium 4, $512 \mathrm{MB}$ DDR e a interface apresentada por meio de uma tela plana de 17'. Para a realização dos testes, os indivíduos fizeram uso de um joystick (controle manual) Microsoft ${ }^{\circledR}$.

O tempo, em segundos, tomado para o indivíduo completar a tarefa foi aferido manualmente por meio de um cronômetro da marca TIMEX ${ }^{\circledR}-$ mo- $^{-}$ delo Triathlon. Os erros ocorridos durante a interação com os ambientes foram aferidos em número absoluto, pelo pesquisador, em uma ficha individual para posterior análise.

\section{Procedimento}

Os participantes foram divididos em três grupos de forma aleatória, de 10 indivíduos cada, e adequadamente posicionados em uma cadeira com encosto e apoio de braços, em frente a uma mesa, segundo a Norma Regulamentadora (NR) n.0 17 - Ergonomia - Redação dada pela Portaria n.0 3.751 de 23 de novembro de 1990 do MT/PS.

Os grupos foram organizados da seguinte forma: o primeiro grupo, considerado como controle, não foi adaptado em nenhum dos ambientes propostos; o segundo grupo treinou no ambiente fechado e o terceiro grupo treinou no ambiente aberto " $\mathrm{A}$ ". A fase de adaptação (treino) ao ambiente, foi caracteri- 
zada pela exposição dos indivíduos a cinco oportunidades seqüenciais de interação (blocos), com intervalos de 2 minutos (repouso) entre cada bloco.

Este tempo de intervalo é defendido por autores (4), em seus estudos, como adequado para a reprodução de uma determinada tarefa, favorecendo o aprendizado na realização da mesma.

Após a fase de adaptação, os dois grupos e o grupo controle foram expostos à interação nos ambientes abertos "B" e "C" sequencialmente, uma única vez.

Todos os participantes receberam as instruções sobre os procedimentos da pesquisa, e as dúvidas foram respondidas pelo pesquisador.

\section{Análise Estatística}

As variáveis dependentes Erro e Tempo para realização das tarefas foram consideradas para análise do desempenho dos voluntários. Os dados obtidos na primeira fase da pesquisa (adaptação), foram submetidos à análise estatística utilizando-se os testes de Komolgorov-Smirnov (determinando a validade da amostra para a pesquisa), Análise de Variância para Medidas Repetidas - (ANOVA) e Análise de Homogeneidade de Variância, (determinando a diferença entre os grupos nas variáveis dependentes Erro e Tempo, nos ambientes propostos), e o NewmanKeuls (um pós-teste utilizado para a verificação de diferenças entre os grupos).

Para a segunda fase da pesquisa (pós adaptação), a análise dos resultados concentrou-se somente na variável Tempo, uma vez que a variável Erro não mostrou valores expressivos entre os grupos, durante a fase de adaptação. A análise foi realizada utilizando-se os testes ANOVA e Newman - Keuls. Foram considerados como significativos os valores de $p<0,05$.

\section{RESULTADOS}

O teste de Komolgorov-Smirnov demonstrou homogeneidade e distribuição normal da amostra em relação a variável Tempo. A análise das tarefas não revelou valores significativos $(p>0,05)$. Neste teste, estes valores justificaram a continuidade da análise, com a validação da amostra.

Houve tendência a uma interação entre os fatores grupo e tarefa $(p=0,099)$. O grupo treinado no ambiente fechado tendeu a melhorar o tempo de desempenho em função das tarefas, o que não foi observado com grupo treinado no ambiente aberto " $\mathrm{A}$ ".

Houve uma diferença significativa entre as tarefas do grupo treinado no ambiente fechado e do grupo treinado no ambiente aberto "A" $(p=0,0036)$.
Durante a fase de adaptação, os indivíduos do grupo treinado no ambiente fechado apresentaram uma média e desvio-padrão de tempo significativamente maior na primeira tarefa $(94,8 \pm 42,80 \mathrm{~s})$ quando comparados a terceira $(77,95 \pm 25,14 \mathrm{~s} ; p=$ $0,028)$, quarta $(73,3 \pm 29,36 \mathrm{~s} ; p=0,007)$, e quinta tarefas $(72,5 \pm 16,79 \mathrm{~s} ; p=0,007)$ de tarefas.

O grupo que durante a fase de adaptação treinou no ambiente fechado, apresentou uma tendência $(p=0,068)$ a levar mais tempo para completar a tarefa dos ambientes abertos "B" (109,8 \pm 49,29 s) e "C" (95,4 $\pm 22,37 \mathrm{~s})$, do que o grupo que se adaptou no ambiente aberto "A", ao executar a tarefa dos mesmos ambientes abertos "B" (86,1 $\pm 17,89$ s) e "C" (91,4 \pm 26,89 s).

Nos ambientes aberto "A" e fechado, o tempo de desempenho dos indivíduos no quinto bloco da fase de adaptação apresentam média e desvio padrão significativamente mais rápido $(72,5 \pm 16,79$ s) quando comparados ao tempo de desempenho nos ambientes abertos "B" (97,95 \pm 38,08 s) e "C" (93,4 \pm 24,16 s).

Os resultados mostram não existir diferença significativa $(p=0,3940)$ entre o desempenho do grupo controle no desempenho das tarefas dos ambientes abertos "B" (104 \pm 28,66 s) e "C" (93,3 \pm $31,24 \mathrm{~s}$ ), com o desempenho dos grupos treinados nos ambientes fechado e aberto "A", mas o grupo controle mostrou um desempenho similar ao do grupo que treinou no ambiente Fechado.

Em relação à variável Erro, a análise de variância para medidas repetidas (ANOVA) mostrou não haver diferença significativa entre os indivíduos dos grupos treinados nos ambientes fechado e aberto "A", nas cinco tarefas realizadas durante a fase de adaptação $(p>0,05)$.

\section{DISCUSSÃO}

Os grupos estudados mostraram melhora progressiva de desempenho a cada tarefa realizada durante a fase de adaptação aos ambientes fechado e aberto "A", diminuindo o tempo de execução da tarefa.

O grupo treinado no ambiente fechado levou um tempo maior na execução da tarefa quando comparado ao grupo que treinou no ambiente aberto "A", e apresentou melhora do desempenho a cada tarefa realizada.

Estes dados em conjunto, sugerem que a prática de tarefas em ambientes fechados é benéfica para a fase de aquisição do aprendizado. Em contrapartida, a prática de tarefas em ambientes abertos é prejudicial para a fase de aquisição, mas benéfica para as fases de retenção e transferência do aprendizado. 
Nas tarefas em ambientes abertos, o indivíduo é obrigado a observar e captar aspectos relevantes da tarefa, melhorando o desempenho.

O nível de interferência contextual e o nível de conhecimento de resultados durante a aquisição de habilidades motoras influenciam a capacidade de retenção do aprendizado e transferência deste aprendizado para o desempenho de tarefas similares, o que pode ser confirmado neste estudo ${ }^{1}$.

Estes resultados demonstram que à medida que o desempenho de uma habilidade melhora, certas características comportamentais do desempenho tornam-se mais estáveis, ou seja, não serão facilmente perturbadas por pequenas alterações nas características pessoais ou ambientais. A interação do indivíduo com o ambiente, pode provocar condições de desequilíbrio, exigindo a auto-organização das respostas motoras para que o mesmo se adapte a estas diferentes situações ${ }^{1,4}$.

Ao interagir com os ambientes, os participantes da pesquisa mostraram variação entre os níveis de desempenho de uma tentativa para outra. As respostas motoras decorrentes da auto-organização exigida para interagir com os ambientes podem tornarse mais consistentes com a prática ou treino da tarefa a ser executada levando a um melhor desempenho ${ }^{6}$.

Neste contexto, a aprendizagem não se limita a um aperfeicoamento do desempenho, mas junto a características relacionadas ao controle motor, podem expressar o comportamento motor e funcional de uma pessoa ${ }^{12}$.

Ao considerarmos os resultados da análise da variável Erro durante a fase de adaptação, não foi observada diferença entre os grupos. Podemos sugerir que os indivíduos não mostraram dificuldades no aprendizado da tarefa, adquirindo comportamentos motores consistentes durante esta fase ${ }^{12}$.

Assim, a aprendizagem pode ser considerada como um aperfeiçoamento do desempenho, ou seja, o desempenho aperfeiçoado permite que o indivíduo se adapte a uma grande variedade de características do contexto ou ambiente de interação ${ }^{13}$.

Tanto a teoria generalizada dos programas motores como a teoria dos efeitos da interferência contextual, baseiam-se no conceito de variabilidade da pratica, relacionando-a com a melhora da retenção e transferência de habilidades motoras ${ }^{14}$.

As alterações do desempenho dos participantes foram observadas na comparação dos tempos dos indivíduos no último bloco da fase de adaptação com os tempos dos indivíduos nos ambientes abertos " $\mathrm{B}$ " e " $\mathrm{C}$ ".
Os resultados mostram que o grupo adaptado no ambiente aberto " $\mathrm{A}$ " teve uma tendência a ser mais rápido, na execução das tarefas dos ambientes abertos "B" e "C", que o grupo adaptado no ambiente fechado. e que não houve diferença significativa quando estes dois grupos foram comparados ao grupo controle, no desempenho das tarefas dos ambientes abertos "B" e "C". O grupo controle mostrou um desempenho similar ao do grupo que treinou no ambiente fechado.

Este cenário nos remete à literatura, onde temos que o conceito de transferência de aprendizado afirma que a influência da experiência prévia na interação do indivíduo com um novo contexto ou na aprendizagem de uma nova habilidade pode ser uma influência positiva, negativa ou neutra ${ }^{12}$.

A transferência positiva ocorre quando a experiência com uma habilidade ajuda ou facilita o desempenho do indivíduo na tarefa. A transferência negativa ocorre quando a experiência com uma habilidade impede ou interfere no desempenho do indivíduo na tarefa. A transferência neutra não altera o desempenho em um novo contexto ou na aprendizagem de uma nova habilidade ${ }^{12}$.

Então, apesar da diferença pouco significativa no tempo de desempenho das tarefas quando comparado aos demais grupos, o grupo adaptado no ambiente aberto "A" foi mais consistente em relação ao comportamento motor e interação com os outros ambientes. A adaptação prévia neste grupo determinou uma transferência positiva do aprendizado.

Essa relação pode explicar ainda, a similaridade observada entre o desempenho nas tarefas do grupo controle e do grupo adaptado ao ambiente fechado.

Assim, vamos de encontro à hipótese desta pesquisa, já que os indivíduos adaptados à execução de tarefas visuo-espaciais que envolviam situações desconhecidas e não orientadas (ambiente aberto) tiveram melhor desempenho nestas tarefas quando comparados à indivíduos adaptados à situações estáveis e orientadas (ambiente fechado).

A interferência contextual (ambiente de interação) foi a maior determinante das estratégias motoras a serem desenvolvidas pelos indivíduos. A prática sob grande interferência contextual determinou uma tendência para o processamento mais elaborado do material aprendido, e desta forma facilitou a retenção de habilidades motoras, tanto quanto a diminuição da dependência da memória no restabelecimento do contexto inicial da ação realizada nos ambientes computacionais propostos ${ }^{8}$. 


\section{CONCLUSÃO}

Este estudo sugere que indivíduos adaptados à execução de tarefas visuo-espaciais que envolvam situações desconhecidas e não orientadas (ambiente aberto) apresentam uma tendência a um melhor desempenho nestas tarefas, quando comparados a indivíduos adaptados em situações estáveis e orientadas (ambiente fechado), na realização de uma mesma tarefa.

Pesquisas futuras são necessárias para avaliar as implicações clínicas dos resultados deste estudo, bem como a relação dos dados obtidos em ambiente computacional, com o ambiente natural.

\section{REFERÊNCIAS BIBLIOGRÁFICAS}

1. Carlson NR. Fisiologia do Comportamento Motor. São Paulo: Editora Manole, 2002, $722 \mathrm{p}$.

2. Jarus T. Motor learning and Occupational Therapy: The Organization of practice. Am J Occup Ther 1994,48(9):138-44.
3. Kamm K, Thelen E, Jensen JL. A Dynamical Sistems Approach to Motor Development. Phys Ther 1990;70:763-75.

4. Trombly CA. Occupational Therapy for Physical Disfunction. 4th ed. Baltmore: Williams \& Wilkins, 1995, 811 p.

5. Scholz JP. Dynamic Pattern Theory - Some Implications for Therapeutics. Phys Ther 1999;70(12):827-42.

6. Kelso S. Advances in Motor Learning. Champaign: Ed. Human Knetics, 1996, 268 p.

7. Keele SW, Posner MI. Processing of Visual Feedback in Rapid Moviments. J Exp Psychol Gen 1968;77(1):155-8.

8. Shea JB, Morgan RL. Contextual Interference Effects on the Acquisition, Retention and Transfer of a Motor Skill. J Exp Psychol Learn Mem Cogn 1979;5(2):179-87.

9. Lee DN, Reddish PE. Plummeting Gannets: A Paradigm of Ecological Optcs. Nature 1981;293(5830):293-4.

10. Warren Jr WH. Perceiving Affordances: Visual Guidence of Stair Climbing. J Exp Psychol Hum Percept Perform 1984;10(3):683-703.

11. Rugy A. The Control of Human Locomotor Point Under Restricted Informational Conditions. Neurosci Lett 2000;281:87-90.

12. Magill RA. Aprendizagem Motora Conceitos e Aplicações. São Paulo: Editora Edgard Blucher ltda., 2002, 369 p.

13. Shumway-Cook A, Woollacott MH. Controle Motor Teorias e Aplicações Práticas. São Paulo: Editora Manole, 2003, 743 p.

14. Schmidt RA. Motor control and learning: a behavioral emphasis. 2nd ed. Champaign: Human Kinetics, 1988, 365 p. 